\title{
UoW: Multi-task Learning Gaussian Process for Semantic Textual Similarity
}

\author{
Miguel Rios \\ Research Group in Computational Linguistics \\ University of Wolverhampton \\ Stafford Street, Wolverhampton, \\ WV1 1SB, UK \\ M.Rios@wlv.ac.uk
}

\begin{abstract}
We report results obtained by the UoW method in SemEval-2014's Task 10 - Multilingual Semantic Textual Similarity. We propose to model Semantic Textual Similarity in the context of Multi-task Learning in order to deal with inherent challenges of the task such as unbalanced performance across domains and the lack of training data for some domains (i.e. unknown domains). We show that the Multi-task Learning approach outperforms previous work on the 2012 dataset, achieves a robust performance on the 2013 dataset and competitive results on the 2014 dataset. We highlight the importance of the challenge of unknown domains, as it affects overall performance substantially.
\end{abstract}

\section{Introduction}

The task of Semantic Textual Similarity (STS) (Agirre et al., 2012) is aimed at measuring the degree of semantic equivalence between a pair of texts. Natural Language Processing (NLP) applications such as Question Answering (Lin and Pantel, 2001), Text Summarisation (Lin and Hovy, 2003) and Information Retrieval (Park et al., 2005) rely heavily on the ability to measure semantic similarity between pairs of texts. The STS evaluation campaign provides datasets that consist of pairs of sentences from different NLP domains such as paraphrasing, video paraphrasing, and machine translation (MT) evaluation. The participating systems are required to predict a graded similarity score from 0 to 5 , where a score of 0 means that the two sentences are on different topics and

This work is licensed under a Creative Commons Attribution 4.0 International Licence. Page numbers and proceedings footer are added by the organisers. Licence details: http://creativecommons.org/licenses/by/4.0/

\author{
Lucia Specia \\ Department of Computer Science \\ University of Sheffield \\ Regent Court, 211 Portobello, \\ Sheffield, S1 4DP, UK \\ L.Specialsheffield.ac.uk
}

a score of 5 means that the two sentences have exactly the same meaning.

Methods for STS are commonly based on computing various types of similarity metrics between the pair of sentences, where the similarity scores are used as features to train regression algorithms. Bär et al. (2012) use similarity metrics of varying complexity. The range of features goes from simple string similarity metrics to complex vector space models. The method yielded the best average results based on the official evaluation metrics, despite not having achieved the best results in all individual domains. Šarić et al. (2012) use a similar set up, extracting features from similarity metrics, where these features are based on wordoverlap and syntax similarity. The method was among the best for domains related to paraphrasing. It also achieved a high correlation between the training and test data. In contrast, for the machine translation data the performance in the test set was lower than the one over the training data. A possible reason for the poor results on this domain is the difference in length between the training and test sentences, as in the test data the pairs tend to be short and share similar words. Šarić et al. (2012) claim that these differences show that the MT training data is not representative of the test set given their choice of features.

Most of the participating systems in the STS challenges achieve good results on certain domains (i.e. STS datasets), but poor results on others. Even the most robust methods still show a big gap in performances for different datasets. In the second evaluation campaign of STS a new challenge was proposed: domains for which no training sets are provided, but only test sets. Heilman and Madnani (2013) propose to incorporate domain adaptation techniques (Daumé et al., 2010) for STS to generalise models to new domains. They add new features into the model, where the feature set contains domain specific features plus 
general task features. The machine learning algorithm infers the extra weights of each specific domain and of the general domain. When an instance of a specific domain is to be predicted, only the copy of the features of that domain will be active; if the domain is unknown, the general features will be active. Severyn et al. (2013) propose to use meta-classification to cope with domain adaptation. They merge each pair into a single text and extract meta-features such as bag-ofwords and syntactic similarity scores. The metaclassification model predicts, for each instance, its most likely domain based on these features.

A possible solution to alleviate unbalanced performances on different domains is to model STS in the context of Multi-task Learning (MTL). The motivation behind MTL is that by learning multiple related tasks simultaneously the model performance may improve compared to the case where the tasks are learnt separately. MTL is based on the assumption that related tasks can be clustered and inter-task correlations between tasks within the same cluster can be transferred.

We propose to model STS using MTL based on a state-of-the-art STS feature set (Šarić et al., 2012). As algorithm we use a non-parametric Bayesian approach, namely Gaussian Processes (GP) (Rasmussen, 2006). We show that the MTL model outperforms previous work on the 2012 datasets and leads to robust performance on the 2013 datasets. On the STS 2014 challenge, our method shows competitive results.

\section{Experimental Setting}

We apply MTL to cope with the challenge of unbalanced performances across domains and unknown domains present in the STS datasets.

\subsection{TakeLab Features}

We use the features from one the top performing system in STS 2012: the TakeLab ${ }^{1}$ system, which is publicly available. It extracts the following types of features:

N-gram overlap is the harmonic mean of the degree of matching between the first and second texts, and vice-versa. The overlap is computed for unigrams, bigrams, and trigrams.

WordNet-augmented word overlap is the partial WordNet path length similarity score as-

\footnotetext{
${ }^{1}$ http://takelab.fer.hr/sts/
}

signed to words that are not common to both texts.

Vector space sentence similarity is the representation of each text as a distributional vector by summing the distributional (i.e., LSA) vectors of each word in the text and taking the cosine distance between these texts vectors.

Shallow NE similarity is the matching between Named Entities (NE) that indicates whether they were found in both texts.

Numbers overlap is an heuristic that penalises differences between numbers in texts.

Altogether, these features make up a vector of 21 similarity scores.

\subsection{Multi-task Gaussian Processes}

Gaussian Processes (Rasmussen, 2006) is a Bayesian non-parametric machine learning framework based on kernels for regression and classification. In GP regression, for the inputs $x$ we want to learn a function $f$ that is inferred from a GP prior:

$$
f(x) \sim G P(m(x), k(x, x \prime)),
$$

where $m(x)$ defines a 0 mean and $k(x, x \prime)$ defines the covariance or kernel functions. In the single output case, the random variables are associated to a process $f$ evaluated at different values of the input $x$. In the multiple output case, the random variables are associated to different processes and evaluated at different values of $x$.

We are interested in the intrinsic coregionalization model for GP. A coregionalization model is a heterotopic MTL model in which each output is associated with a different set of inputs. In our case the different set of inputs are the STS domains (i.e. datasets). The intrinsic coregionalization model (i.e. MTL-GP) is based on a separable multi-task kernel (Álvarez et al., 2012) of the form

$$
K(X, X)=B \otimes k(X, X),
$$

where $k(X, X)$ is a standard kernel over the input points and $B$ is a positive semi-definite matrix encoding task covariances, called coregionalization matrix. $B$ is built from other matrices $B=W W^{\top}+\operatorname{diag}(k)$, where $W$ is a matrix that determines the correlations between the different outputs and $k$ is a matrix which allows the outputs 
(i.e. tasks) to behave independently. The representation of data points is augmented with task ids and given the id of a pair of data points the covariance from the standard kernel between them is multiplied by a corresponding covariance from $B$, which modifies the data points' covariance depending on whether they belong to the same task or different tasks.

The coregionalization matrix $B$ allows us to control the amount of inter and intra task transfer of learning among tasks. Cohn and Specia (2013) propose different types of $B$ matrices to model the problem of predicting the quality of machine translations. They developed $B$ matrices that represent an explicit intra-task transfer to be a part of the parameterised kernel function. We use a default $B$ where the weights of the matrix are learnt along with the hyper-parameters by the GP tool.

For training our method we use the GPy toolkit ${ }^{2}$ with a combination of RBF and coregionalization kernels. The parameters used to build the coregionalization matrix are the number of outputs to coregionalize and the rank of $W$. For example, in the 2012 training set, the number of outputs to coregionalize is 3 , given that we have three tasks/domains. The $B$ matrix and the RBF kernel hyper-parameters are jointly optimised. Each instance of the training data is then augmented with the id of their corresponding task. During testing a new instance has to be matched to a specific task/domain id from the training data. In the case of an unknown test domain, we match it to a training domain which is similar, given the description of the test dataset.

For the STS 2014 dataset, given the large number of training instances, we train a sparse GP model within GPy. The main limitation of the GP model is the that memory demands grow $O\left(n^{2}\right)$, and the computational demands grow $O\left(n^{3}\right)$, with $n$ equals the number of training instances. Sparse methods (e.g. (Titsias, 2009)) try to overcome this limitation by constructing an approximation of the full model on a smaller set of $m$ support or inducing instances that allow the reduction of computational demands to $O\left(n m^{2}\right)$. For the sparse GP we use the same combination of kernels as the full model, where we chose empirically the number of inducing instances $m$ and the GP tool randomly selects the instances from the training data.

\footnotetext{
${ }^{2}$ https://github.com/SheffieldML/GPy
}

\section{Results and Discussion}

In what follows we show a comparison with previous work on the STS 2012 and 2013 datasets, and the official results for English and Spanish STS 2014 datasets.

\subsection{STS 2012 and STS 2013}

For training we use the STS 2012 training datasets and we compare the results on the STS 2012 with publicly available systems and with the official Baseline, which is based on the cosine metric computed over word overlaps. The official evaluation metric is Pearson's correlation. We match the unknown domain OnWN to MSRpar given that the domain of paraphrasing is that of news from the web, which potentially contains a broad enough vocabulary to cover OnWN.

Table 3.1 shows a comparison of the MTL-GP with previous work on the STS 2012 data, where our method outperforms them for most of the domains. Our method improves the results of TakeLab with the same feature set. In other words, the transfer learning improves over (Šarić et al., 2012), which is trained with a separate Support Vector Regression model for each domain. We note that we can only compare our method against the simpler version of TakeLab that is available. A different version using syntactic features was also proposed, where most results do not show a significant variation, except for an improvement of $\mathrm{r}=0.4683$ in the SMTnews dataset. For the complete alternative results we refer the reader to (Šarić et al., 2012).

On the STS 2013 dataset, we compare our method with work based on domain adaptation and the official baseline. We use the 2012 data for training as no additional training data is provided in 2013. Table 3.1 shows all the possible matching combinations between the STS 2013 test sets and STS 2012 training sets. The best results are given by matching the STS 2013 test sets with the MSRvid domain, where all 2013 sets achieve their best results.

In Table 3.1, we show the comparison with previous work on the 2013 datasets, where we use the best matching result from Table 3.1 (MSRvid). Our method shows very competitive results but only with the correct matching of domains, whereas the worst performed matching (SMTeuroparl, Table 3.1) shows results that are closer to the official Baseline. In previous work 


\begin{tabular}{cccccc}
\hline \hline Method & MSRpar & MSRvid & SMTeuroparl & SMTnews & OnWN \\
\hline Šarić et al. (2012) & $\mathbf{0 . 7 3 4 3}$ & 0.8803 & 0.4771 & 0.3989 & 0.6797 \\
Bär et al. (2012) & 0.68 & 0.8739 & 0.5280 & 0.4937 & 0.6641 \\
MTL-GP & 0.7324 & $\mathbf{0 . 8 8 7 7}$ & $\mathbf{0 . 5 6 1 5}$ & $\mathbf{0 . 6 0 5 3}$ & $\mathbf{0 . 7 2 5 6}$ \\
Baseline & 0.4334 & 0.2996 & 0.4542 & 0.3908 & 0.5864 \\
\hline
\end{tabular}

Table 1: Comparison with previous work on the STS 2012 test datasets.

(Heilman and Madnani, 2013), domain adaptation is performed with the addition of extra features and the subsequent extra parameters to the model, where in the MTL-GP the transfer learning is done with the coregionalization matrix and does not depend on large amounts of data.

\subsection{English STS 2014}

The training dataset consists of the combination of each English training and test STS datasets from 2012 and 2013, which results in 7 domains. For testing, in our first run we matched similar domains with each other and the unknown domain with MSRpar. For our second run, we matched the unknown domains with a similar one. The domain matching (test/training) was done as follows: deft-forum/MSRpar, deft-news/SMTnews, tweet-news/SMTnews and images/MSRvid. For our third run, the difference in matching is for deftnews/headlines and tweet-news/headlines, where the other domains remain with the same matching. Table 3.2 shows the official STS 2014 results where our best method (i.e. run3) achieves rank 10.

In Table 3.2, we show the comparison of the MTL-GP and the sparse MTL-GP with the best 2014 system (DLSCU run2). For both MTL methods we match the 2014 domains with the training domain headlines. For the sparse MTL-GP, we chose empirically a number $m$ of 500 randomly induced points. For reference, the correlation of sparse MTL-GP with 50 points on deftforum is $\mathrm{r}=0.4691$ obtained in 0.23 hours, with 100 points, $r=0.4895$, with 500 points, $r=0.4912$, and with 1000 points, $r=0.4911$. The sparse MTL-

\begin{tabular}{|c|c|c|c|}
\hline Test $\quad$ Train & MSRvid & MSRpar & SMTeuroparl \\
\hline Headlines & 0.6666 & 0.6595 & 0.5693 \\
\hline OnWN & 0.6516 & 0.4635 & 0.4113 \\
\hline FNWN & 0.4062 & 0.3217 & 0.2344 \\
\hline
\end{tabular}

Table 2: Matching of new 2013 domains with 2012 training data.
GP with 500 points runs in 1.38 hours, compared to 2.39 hours for the full MTL-GP ${ }^{3}$. Additionally, the sparse version achieves similar results to the full model and very competitive performance compared to the best STS 2014 system. However, the result for OnWN is substantially lower than the best system. This result can be highly improved $(\mathrm{r}=0.7990)$ if the test set is matched with the correspondent training domain.

\subsection{Spanish STS 2014}

For the Spanish STS subtask we use both simple and state-of-the-art (SoA) features to train the MTL-GP. The simple features are similarity scores from string metrics such as Levenshtein, Gotoh, Jaro, etc. ${ }^{4}$ The SoA similarity features come again from TakeLab. The training dataset consists of the combination of each English STS domains from 2012 and 2013 and the Spanish trial dataset with task-id matching each instance to a given domain. We represent the feature vectors with sparse features for the English and Spanish training datasets, where in English the pairs have simple and SoA features, and for Spanish, only the simple features. In other words, the feature vectors have the same number of features (34): 13 simple features and 21 SoA features. However, for Spanish the SoA features are set to 0 in training and testing. The motivation to use SoA and simple features in English is that the extra information will improve the transfer learning on the English domains and discriminate between the English domains and the Spanish domain, which only contains simple features. For testing we only extracted the simple features; the SoA features were set to 0 . For the coregionalization matrix we set the number of domains to be the English STS domains from 2012 and 2013, plus the Spanish trial, where the Spanish is treated as an additional domain, which results in 8 domains. In the first run of testing, we matched the test datasets to the Spanish domain, and in the second run we matched the datasets to the English MSRpar do-

\footnotetext{
${ }^{3}$ Intel Xeon(R) at $2.67 \mathrm{GHz}$ with 24 cores

${ }^{4}$ https://github.com/Simmetrics/simmetrics
} 


\begin{tabular}{cccc}
\hline \hline Method & Headlines & OnWN & FNWN \\
\hline Heilman and Madnani (2013) & $\mathbf{0 . 7 6 0 1}$ & 0.4631 & 0.3516 \\
Severyn et al. (2013) & 0.7465 & 0.5572 & 0.3875 \\
MTL-GP & 0.6666 & $\mathbf{0 . 6 5 1 6}$ & $\mathbf{0 . 4 0 6 2}$ \\
Baseline & 0.5399 & 0.2828 & 0.2146 \\
\hline
\end{tabular}

Table 3: Comparison between best matching MTL-GP (MSRvid) and previous work on the STS 2013 test datasets.

\begin{tabular}{ccccccccc}
\hline \hline Run & deft-forum & deft-news & headlines & images & OnWN & tweet-news & Weighted mean & rank \\
\hline UoW run1 & 0.3419 & 0.7512 & 0.7535 & 0.7763 & 0.7990 & 0.7368 & 0.7143 & 11 \\
UoW run2 & 0.3419 & 0.5875 & 0.7535 & 0.7877 & 0.7990 & 0.6281 & 0.6817 & 17 \\
UoW run3 & 0.3419 & 0.7634 & 0.7535 & 0.7877 & 0.7990 & 0.7529 & 0.7207 & 10 \\
\hline
\end{tabular}

Table 4: Official English STS 2014 results.

main. Table 3.3 shows the official results for the Spanish subtask, where our method achieves competitive performance, placed 7 in the systems ranking. We only show the results for the first run as both runs achieved the same performance.

\begin{tabular}{lllll}
\hline \hline Run & Wikipedia & News & $\begin{array}{l}\text { Weighted } \\
\text { mean }\end{array}$ & rank \\
\hline UoW & 0.7483 & 0.8001 & 0.7792 & 7 \\
\hline
\end{tabular}

Table 6: Official Spanish STS 2014 results.

Table 3.3 shows the comparison of the best Spanish STS 2014 system (UMCC_DLSI run2) against two different sparse MTL-GP matched with the Spanish trial with 500 inducing points. Sparse MTL-GP run1 uses the sparse features described above, while run2 uses a modification of the feature set consisting in specific features for each type of domain. For the English domains the simple features are set to 0 , and for Spanish the SoA are still set to 0 . The difference between sparse MTL-GP models is very small, where the use of all the features on the English domains improves the results. However, the performance of both models is still substantially lower than that of the best system.

\begin{tabular}{ccc}
\hline \hline Run & Wikipedia & News \\
\hline UMCC_DLSI run2 & 0.7802 & 0.8254 \\
Sparse MTL-GP run1 & 0.7468 & 0.7959 \\
Sparse MTL-GP run2 & 0.7380 & 0.7878 \\
\hline
\end{tabular}

Table 7: Comparison of best system against sparse MTL-GP STS 2014 results.

\section{Conclusions}

We propose the use of MTL for STS. We show that MTL improves the results of one of the best STS systems, TakeLab. However, the match- ing of an unknown domain during testing proved a key challenge that affects performance significantly. Given the results of STS 2013 and 2014, our method tends to achieve best results when known/unknown domains are matched to the same training domains (i.e. MSRpar for 2013 and headlines for 2014). The sparse MTL-GP shows similar performance to the full GP model, but takes half the time to be trained. In the Spanish subtask, we train our method with English datasets and the Spanish trial data as an additional domain. For this subtask our method also shows competitive results. Future work involves the automatic matching of unknown domains at test time via metaclassification (Severyn et al., 2013).

\section{Acknowledgments}

This work was supported by the Mexican National Council for Science and Technology (CONACYT), scholarship reference 309261, and by the QTLaunchPad (EU FP7 CSA No. 296347) project.

\section{References}

Eneko Agirre, Mona Diab, Daniel Cer, and Aitor Gonzalez-Agirre. 2012. Semeval-2012 task 6: A pilot on semantic textual similarity. In Proceedings of the First Joint Conference on Lexical and Computational Semantics, SemEval '12, pages 385-393, Stroudsburg, PA, USA.

Mauricio A. Álvarez, Lorenzo Rosasco, and Neil D. Lawrence. 2012. Kernels for vector-valued functions: A review. Found. Trends Mach. Learn., 4(3):195-266, March.

Daniel Bär, Chris Biemann, Iryna Gurevych, and Torsten Zesch. 2012. Ukp: Computing semantic textual similarity by combining multiple content similarity measures. In Proceedings of the First 


\begin{tabular}{ccccccc}
\hline \hline Run & deft-forum & deft-news & headlines & images & OnWN & tweet-news \\
\hline DLSCU run2 & 0.4828 & $\mathbf{0 . 7 6 5 7}$ & $\mathbf{0 . 7 6 4 6}$ & $\mathbf{0 . 8 2 1 4}$ & $\mathbf{0 . 8 5 8 9}$ & $\mathbf{0 . 7 6 3 9}$ \\
Best matching MTL-GP & 0.4903 & 0.7633 & 0.7535 & 0.8063 & 0.7222 & 0.7528 \\
Sparse MTL-GP & $\mathbf{0 . 4 9 1 0}$ & 0.7642 & 0.7540 & 0.8057 & 0.7276 & 0.7539 \\
\hline
\end{tabular}

Table 5: Comparison between best matching MTL-GP (headlines), Sparse MTL-GP and best STS 2014 system.

Joint Conference on Lexical and Computational Semantics, SemEval '12, pages 435-440, Stroudsburg, PA, USA.

Trevor Cohn and Lucia Specia. 2013. Modelling annotator bias with multi-task gaussian processes: An application to machine translation quality estimation. In 51st Annual Meeting of the Association for Computational Linguistics, ACL-2013, pages 3242, Sofia, Bulgaria.

Hal Daumé, III, Abhishek Kumar, and Avishek Saha. 2010. Frustratingly easy semi-supervised domain adaptation. In Proceedings of the 2010 Workshop on Domain Adaptation for Natural Language Processing, DANLP 2010, pages 53-59, Stroudsburg, PA, USA.

Michael Heilman and Nitin Madnani. 2013. Henrycore: Domain adaptation and stacking for text similarity. In Second Joint Conference on Lexical and Computational Semantics (*SEM), pages 96-102, Atlanta, Georgia, USA, June.

Chin-Yew Lin and Eduard Hovy. 2003. Automatic evaluation of summaries using n-gram cooccurrence statistics. In Proceedings of the 2003 Conference of the North American Chapter of the Association for Computational Linguistics on $\mathrm{Hu}$ man Language Technology - Volume 1, NAACL '03, pages 71-78, Stroudsburg, PA, USA.

Dekang Lin and Patrick Pantel. 2001. Discovery of inference rules for question-answering. Nat. Lang. Eng., 7(4):343-360.

Eui-Kyu Park, Dong-Yul Ra, and Myung-Gil Jang. 2005. Techniques for improving web retrieval effectiveness. Inf. Process. Manage., 41(5):1207-1223.

Carl Edward Rasmussen. 2006. Gaussian processes for machine learning. MIT Press.

Aliaksei Severyn, Massimo Nicosia, and Alessandro Moschitti. 2013. ikernels-core: Tree kernel learning for textual similarity. In Second Joint Conference on Lexical and Computational Semantics (*SEM), pages 53-58, Atlanta, Georgia, USA, June.

Michalis Titsias. 2009. Variational Learning of Inducing Variables in Sparse Gaussian Processes. In the 12th International Conference on Artificial Intelligence and Statistics (AISTATS).

Frane Šarić, Goran Glavaš, Mladen Karan, Jan Šnajder, and Bojana Dalbelo Bašić. 2012. Takelab: Systems for measuring semantic text similarity. In Proceedings of the First Joint Conference on Lexical and Computational Semantics, SemEval '12, pages 441448, Stroudsburg, PA, USA. 